\title{
A DUAL-RECIPROCITY BOUNDARY ELEMENT METHOD FOR STEADY INFILTRATION PROBLEMS
}

\author{
I. SOLEKHUDIN ${ }^{\otimes 1,2}$ and K. C. $\mathrm{ANG}^{1}$
}

(Received 7 June, 2012; revised 26 March, 2013)

\begin{abstract}
Steady water infiltration in homogeneous soils is governed by the Richards equation. This equation can be studied more conveniently by transforming to a type of Helmholtz equation. In this study, a dual-reciprocity boundary element method (DRBEM) is employed to solve the Helmholtz equation numerically. Using the solutions obtained, numerical values of the suction potential are then computed. The proposed method is tested on problems involving infiltration from different types of periodic channels in a homogeneous soil. Moreover, the method is also examined using infiltration from periodic trapezoidal channels in three different types of homogeneous soil.
\end{abstract}

2010 Mathematics subject classification: 76M15.

Keywords and phrases: Richards equation, suction potential, dual-reciprocity boundary element method, periodic identical irrigation channels.

\section{Introduction}

Raising crops on farms or plantations where rainfall is low often requires irrigation systems. Crops are usually planted or grown in rows with shallow furrows between these rows. Water supplied to the crops through these furrows should be in sufficient quantity and the soil should be sufficiently moist. In addition, it should be ensured that the plants can easily absorb the water from the soil.

In any irrigation system, it is always desirable to maintain a sufficiently high level of moisture content in the soil so that plant roots are able to extract water or moisture from the soil efficiently. Moisture content in the soil is governed by its moisture potential, which is defined as the potential of forces arising from local interaction between solid and water [10] and often denoted by $\psi$. Many studies of water infiltration problems in irrigation channels involve investigation of the behaviour, characteristics or variation of $\psi$ in various physical situations and contexts.

\footnotetext{
${ }^{1}$ Mathematics and Mathematics Education, National Institute of Education, Nanyang Technological University, 1 Nanyang Walk, Singapore 637616, Singapore; e-mail: kengcheng.ang @nie.edu.sg.

${ }^{2}$ Department of Mathematics, Faculty of Mathematics and Natural Sciences, Gadjah Mada University, Yogyakarta 55281, Indonesia; e-mail: imam.solekhudin@stdmail.nie.edu.sg, imams@ugm.ac.id.

(C) Australian Mathematical Society 2013, Serial-fee code 1446-1811/2013 \$16.00
} 
Infiltration problems from single or periodic channels have been examined by researchers including Azis et al. [4], Batu [5], Clements et al. [8] and Lobo et al. [9]. However, these problems were solved analytically only for very simple cases, such as those considered by Batu. For more realistic, and hence more complicated cases, numerical methods may be needed. Moreover, although analytical methods may be regarded as successful to a certain extent, it is still useful to develop alternative numerical approaches. One approach worth considering is the dual-reciprocity boundary element method (DRBEM), which is known for its flexibility and accuracy in dealing with boundary conditions in many problems.

In this paper, a DRBEM is proposed for obtaining the numerical values of $\psi$ from the steady infiltration from periodic identical irrigation channels. The method is applied to examine infiltration from periodic flat and nonflat channels. The periodic nonflat channels considered here are rectangular, semi-circular, and trapezoidal. Moreover, problems involving infiltration from periodic trapezoidal channels in three different varieties of soils are also examined.

\section{Problem formulation}

We consider an array of equally spaced identical irrigation channels. For every unit length, the surface area of the channels is $2 L$ square units. The distance between the centres of two consecutive channels is $2(L+D)$. We assume that the channels are sufficiently long and that there is a large number of such channels. Hence the flow pattern is two dimensional and the influence of outer channels is negligible. The fluxes on the channels and on the soil surface outside the channels are $v_{0}$ and 0 respectively. The geometries described are as shown in Figure 1.

In view of the symmetry of the problem, it is sufficient to consider the semi-infinite region defined by $0 \leq X \leq L+D$ and $0 \leq Z \leq \infty$. This region is denoted by $R$ with boundary $C$. The usual assumption that there is no flux across $X=0$ and $X=L+D$ is made.

\section{Basic equations}

In the study of steady infiltration problems, a mathematical model that is often employed is the nonlinear partial differential equation

$$
\frac{\partial}{\partial X}\left(K \frac{\partial \psi}{\partial X}\right)+\frac{\partial}{\partial X}\left(K \frac{\partial \psi}{\partial Z}\right)=\frac{\partial K}{\partial Z},
$$

where $K$ is the hydraulic conductivity and $Z$ is the vertical physical space coordinate, pointing positively downward [11-13].

Equation (3.1) can be studied more easily by transforming it to a linear partial differential equation. The Kirchhoff transformation

$$
\Theta=\int_{-\infty}^{\psi} K d \psi
$$




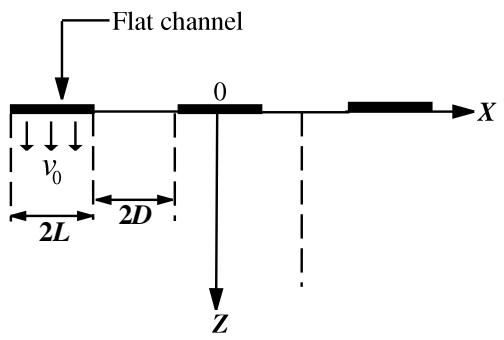

(a) Geometry of periodic flat channels

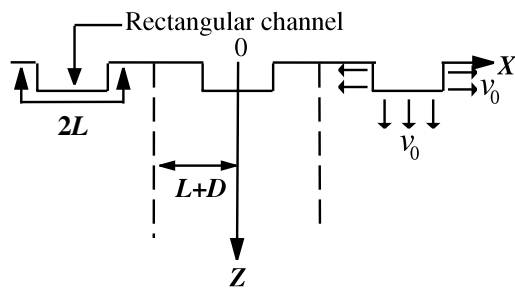

(c) Geometry of periodic rectangular channels

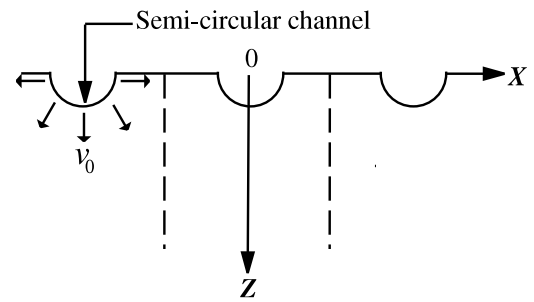

(b) Geometry of periodic semi-circular channels

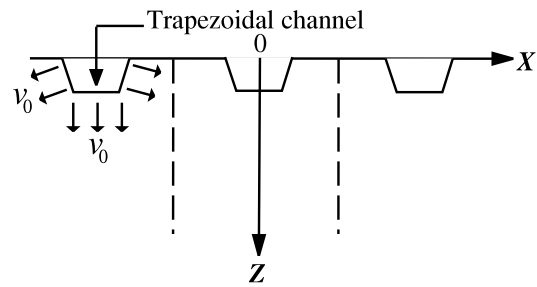

(d) Geometry of periodic trapezoidal channels

FIGURE 1. Geometries of four different periodic channels.

where $\Theta$ is the matric flux potential, and the exponential relation

$$
K(\psi)=K_{s} e^{\alpha \psi}, \quad \alpha>0, \psi \leq 0,
$$

where $K_{s}$ is the saturated hydraulic conductivity and $\alpha$ is an empirical parameter [5, 11-13], yield

$$
\alpha \Theta=K_{s} e^{\alpha \psi} .
$$

Using equations (3.2) and (3.3), equation (3.1) may be transformed to the linear equation

$$
\frac{\partial^{2} \Theta}{\partial X^{2}}+\frac{\partial^{2} \Theta}{\partial Z^{2}}=\alpha \frac{\partial \Theta}{\partial Z}
$$

The horizontal and vertical components of the flux, which are functions of the matric flux potential, are

$$
U=-\frac{\partial \Theta}{\partial X} \quad \text { and } \quad V=\alpha \Theta-\frac{\partial \Theta}{\partial Z}
$$

respectively [5]. The flux normal to the surface with outward pointing normal $\mathbf{n}=\left(n_{1}, n_{2}\right)$ is given by

$$
F=-\frac{\partial \Theta}{\partial X} n_{1}+\left(\alpha \Theta-\frac{\partial \Theta}{\partial Z}\right) n_{2}
$$


Using the dimensionless variables

$$
x=\frac{\alpha}{2} X, \quad z=\frac{\alpha}{2} Z, \quad \Phi=\frac{\pi \Theta}{v_{0} L}, \quad u=\frac{2 \pi}{v_{0} \alpha L} U, \quad v=\frac{2 \pi}{v_{0} \alpha L} V, \quad f=\frac{2 \pi}{v_{0} \alpha L} F
$$

and the substitution

$$
\Phi=\phi e^{z}
$$

in equation (3.4), we obtain a more convenient equation,

$$
\frac{\partial^{2} \phi}{\partial x^{2}}+\frac{\partial^{2} \phi}{\partial z^{2}}=\phi
$$

which is a type of Helmholtz equation. The dimensionless flux is

$$
f=-e^{z}\left(\frac{\partial \phi}{\partial n}-\phi n_{2}\right)
$$

which yields

$$
\frac{\partial \phi}{\partial n}=\phi n_{2}-e^{-z} f
$$

Fluxes at the surfaces of the channels are $v_{0}$, and therefore dimensionless fluxes are $2 \pi / \alpha L$. This implies that on the surfaces of the channels,

$$
\frac{\partial \phi}{\partial n}=\frac{2 \pi}{\alpha L} e^{-z}+\phi n_{2}
$$

The condition of zero flux across the soil surface outside the channels, and along $X=0$ and $X=L+D$, implies

$$
\frac{\partial \phi}{\partial n}=-\phi
$$

on the surface of the soil outside the channels,

$$
\frac{\partial \phi}{\partial n}=0, \quad x=0 \text { and } z \geq 0,
$$

and

$$
\frac{\partial \phi}{\partial n}=0, \quad x=\frac{\alpha}{2}(L+D) \text { and } z \geq 0 .
$$

It is assumed that as $X^{2}+Z^{2} \rightarrow \infty, \partial \Theta / \partial X=0$ and $\partial \Theta / \partial Z=0$ (see the article by Batu [5]). Thus

$$
f=2 \phi e^{z}
$$

Hence, using equation (3.6),

$$
\frac{\partial \phi}{\partial n}=-\phi, \quad 0 \leq x \leq \frac{\alpha}{2}(L+D) \text { and } z=\infty .
$$

A DRBEM is employed to obtain numerical solutions to equation (3.5) subject to the boundary conditions (3.7)-(3.11). The DRBEM was initially proposed by Brebbia 
and Nardini [6]. This method has now been used by other researchers, including Ang and Ang [2, 3] and Zhu et al. [14], for various boundary value problems. An integral equation for solving equation (3.5) is

$$
\begin{aligned}
\lambda(\xi, \eta) \phi(\xi, \eta)=\iint_{R} \varphi(x, z ; \xi, \eta) \phi(x, z) d x d z \\
\quad+\int_{C}\left[\phi(x, z) \frac{\partial}{\partial n}(\varphi(x, z ; \xi, \eta))-\varphi(x, z ; \xi, \eta) \frac{\partial}{\partial n}(\phi(x, z))\right] d s(x, z),
\end{aligned}
$$

where

$$
\varphi(x, y ; \xi, \eta)=\frac{1}{4 \pi} \ln \left[(x-\xi)^{2}+(y-\eta)^{2}\right]
$$

is the fundamental solution of the two-dimensional Laplace equation and

$$
\lambda(\xi, \eta)= \begin{cases}\frac{1}{2} & \text { if }(\xi, \eta) \text { lies on the smooth part of } C \\ 1 & \text { if }(\xi, \eta) \in R\end{cases}
$$

The integral equation (3.12) may be solved numerically. The boundary $C$ is discretized by a number of line segments joined end to end, and a number of interior points are chosen as collocation points. The midpoints of all segments are chosen as collocation points on the boundary. Let $N$ and $M$ be the numbers of the line segments and the interior points, respectively. Let $C^{(1)}, C^{(2)}, \ldots, C^{(N)}$ denote the line segments, and $\left(a^{(i)}, b^{(i)}\right)$ the midpoint of $C^{(i)}, i=1,2, \ldots, N$. Let $\left(a^{(N+1)}, b^{(N+1)}\right),\left(a^{(N+2)}, b^{(N+2)}\right), \ldots,\left(a^{(N+M)}, b^{(N+M)}\right)$ denote the interior points. The value of $\phi$ at $\left(a^{(n)}, b^{(n)}\right), n=1,2, \ldots, N+M$, which is denoted by $\phi^{(n)}$, may be approximated by

$$
\lambda\left(a^{(n)}, b^{(n)}\right) \phi^{(n)}=\sum_{k=1}^{N+M} v^{(n k)} \phi^{(k)}+\sum_{m=1}^{N}\left[\phi^{(k)} F_{1}^{(m)}\left(a^{(n)}, b^{(n)}\right)-p^{(k)} F_{2}^{(m)}\left(a^{(n)}, b^{(n)}\right)\right],
$$

$n=1,2, \ldots, N+M$, where

$$
\begin{aligned}
v^{(n k)} & =\sum_{i=1}^{N+M} \Upsilon\left(a^{(n)}, b^{(n)} ; a^{(i)}, b^{(i)}\right) \omega^{(i k)}, \\
F_{1}^{(m)}\left(a^{(n)}, b^{(n)}\right) & =\int_{C^{(m)}} \varphi\left(x, z ; a^{(n)}, b^{(n)}\right) d s(x, z), \\
F_{2}^{(m)}\left(a^{(n)}, b^{(n)}\right) & =\int_{C^{(m)}} \frac{\partial}{\partial n}\left(\varphi\left(x, z ; a^{(n)}, b^{(n)}\right)\right) d s(x, z) .
\end{aligned}
$$


The quantities $\Upsilon\left(a^{(n)}, b^{(n)} ; a^{(i)}, b^{(i)}\right)$ and $\omega^{(i k)}$ are defined as

$$
\begin{gathered}
\Upsilon\left(a^{(n)}, b^{(n)} ; a^{(i)}, b^{(i)}\right)=\lambda\left(a^{(n)}, b^{(n)}\right) \chi\left(a^{(n)}, b^{(n)} ; a^{(i)}, b^{(i)}\right) \\
+\left.\sum_{j=1}^{N} \frac{\partial}{\partial n}\left(\chi\left(x, z ; a^{(i)}, b^{(i)}\right)\right)\right|_{(x, z)=\left(a^{(j)}, b^{(j)}\right)} F_{1}^{(j)}\left(a^{(n)}, b^{(n)}\right) \\
+\sum_{j=1}^{N} \chi\left(a^{(j)}, b^{(j)} ; a^{(i)}, b^{(i)}\right) F_{2}^{(j)}\left(a^{(n)}, b^{(n)}\right), \\
{\left[\omega^{(i k)}\right]=\left[\rho\left(a^{(k)}, b^{(k)} ; a^{(i)}, b^{(i)}\right)\right]^{-1},}
\end{gathered}
$$

where

$$
\begin{aligned}
& \rho\left(x, z ; a^{(i)}, b^{(i)}\right)=1+\left(r\left(x, z ; a^{(i)}, b^{(i)}\right)\right)^{2}+\left(r\left(x, z ; a^{(i)}, b^{(i)}\right)\right)^{3}, \\
& \chi\left(x, z ; a^{(i)}, b^{(i)}\right)=\frac{1}{4}\left(r\left(x, z ; a^{(i)}, b^{(i)}\right)\right)^{2}+\frac{1}{16}\left(r\left(x, z ; a^{(i)}, b^{(i)}\right)\right)^{4}+\frac{1}{25}\left(r\left(x, z ; a^{(i)}, b^{(i)}\right)\right)^{5}, \\
& r\left(x, z ; a^{(i)}, b^{(i)}\right)=\sqrt{\left(x-a^{(i)}\right)^{2}+\left(z-b^{(i)}\right)^{2}} .
\end{aligned}
$$

From the numerical values of $\phi$ obtained, the suction potential $\psi$ is computed using

$$
\psi=\frac{1}{\alpha} \ln \left(\frac{\alpha v_{0} L \phi e^{z}}{\pi K_{s}}\right) .
$$

\section{Results and discussion}

The method described above is tested on problems involving infiltration from four different types of periodic identical irrigation channels in Pima clay loam (PCL). The values of both $L$ and $D$ are set to be $50 \mathrm{~cm}$. For PCL, the values of $\alpha$ and $K_{s}$ are $0.014 \mathrm{~cm}^{-1}$ and $9.9 \mathrm{~cm} \mathrm{~d}^{-1}$, respectively $[1,7]$.

The channels with depth are created in such a way that their width is the same, as well as their surface area in contact with water. Using the value of $L$ stated above, the width of the channels is $200 / \pi \mathrm{cm}$, and the depths of the semi-circular and the rectangular channels are $100 / \pi \mathrm{cm}$ and $(50-100 / \pi) \mathrm{cm}$, respectively. For trapezoidal channels, the depth is set to be $75 / \pi \mathrm{cm}$.

The DRBEM described in the preceding section is used to obtain numerical values of $\phi$, and then, using equation (3.13), $\psi$ is obtained. Implementing the DRBEM requires the solution domain to be bounded by a simple closed curve. In this case, we fix it to be between $z=0$ and $z=c$, where $c$ is a positive real number. Following several computational experiments, it was found that setting $c=4$ is sufficient for conditions to be applied at this boundary without any significant impact on the values of $\psi$ in the domain.

For flat channels, we set $N=200$ and $M=625$, and for rectangular channels, $N=203$ and $M=625$. The values of $N$ and $M$ for semi-circular channels are $N=201$ and $M=620$, and for trapezoidal channels, $N=202$ and $M=619$. The variation of $\psi$ 

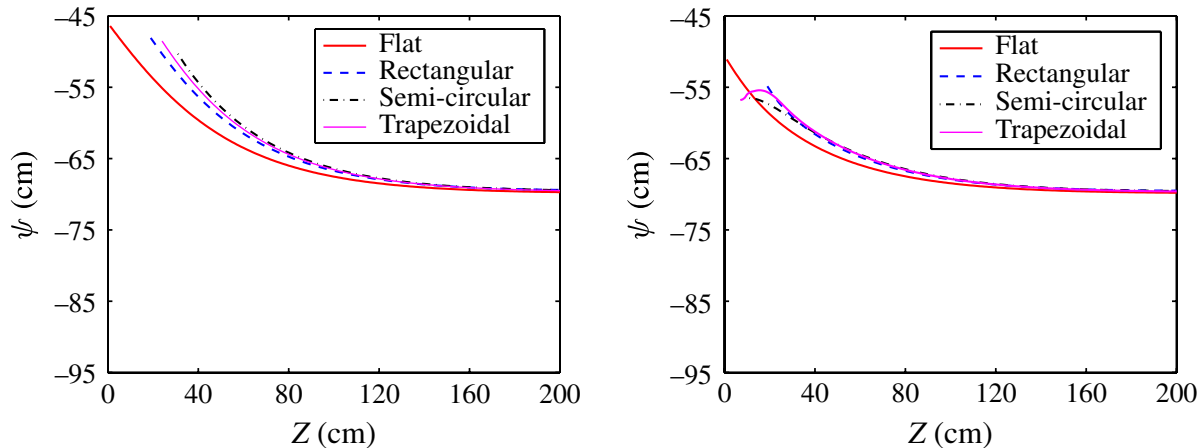

(a) $X=10 \mathrm{~cm}$

(b) $X=30 \mathrm{~cm}$
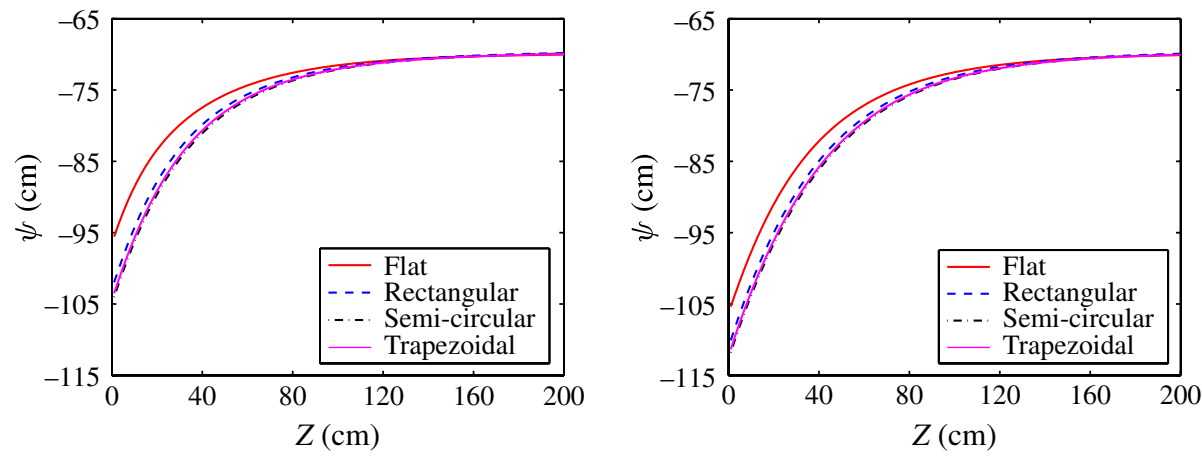

(c) $X=70 \mathrm{~cm}$

(d) $X=90 \mathrm{~cm}$

FigURE 2. Suction potential from four different types of channels at selected values of $X$.

with depth $(Z)$ at different positions $(X)$ from the centres of the channels is presented graphically in Figure 2.

From Figures 2(a) and (b), it is seen that at $X=10 \mathrm{~cm}$ and $X=30 \mathrm{~cm}$, locations under the channel, the values of $\psi$ decrease with $Z$. This indicates that the water content at the shallow level of soil is higher than that at the deeper part. This observation is expected. There are no significant differences in the values of $\psi$ for the three different types of nonflat channels. However, there are differences in $\psi$ when comparing flat channels with the other types of channels, especially at the shallow level of soil. The value of $\psi$ from flat channels is lower than from the other three channels. This may be caused by the positions of the channels. For flat channels, the surface of the channels is on the surface of the soil, that is, at $Z=0 \mathrm{~cm}$. The surfaces of the other three channels are from $Z=0 \mathrm{~cm}$ to a certain level of soil depth.

Away from the channels, at $X=70 \mathrm{~cm}$ and $X=90 \mathrm{~cm}$, the values of $\psi$ are increasing with $Z$ (Figures 2(c) and (d)). This means that the water content at the surface of the soil is the lowest among all levels of soil depth. This is because we have assumed 

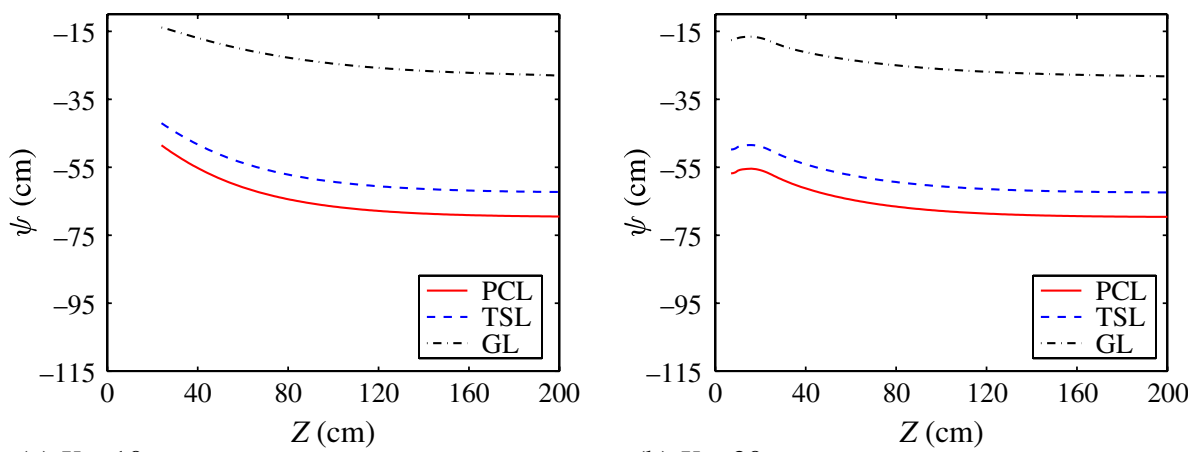

(a) $X=10 \mathrm{~cm}$

(b) $X=30 \mathrm{~cm}$
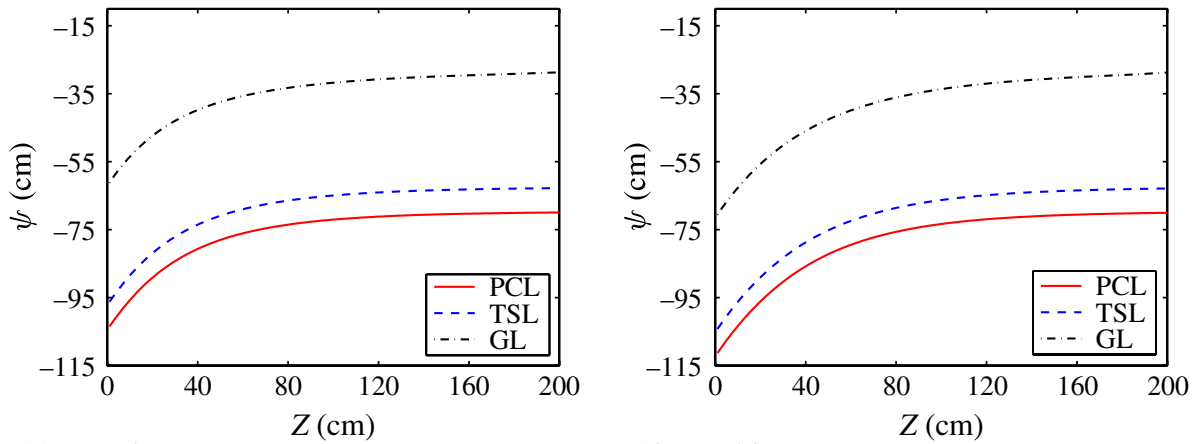

(c) $X=70 \mathrm{~cm}$

(d) $X=90 \mathrm{~cm}$

FIGURE 3. Suction potential from trapezoidal channels in different types of homogeneous soil at selected values of $X$.

that outside the channels, water does not flow into the soil. As before, there is no significant difference in $\psi$ among the three types of nonflat channels, but there are remarkable differences in $\psi$ between flat channels and other channels, particularly at the surface of the soil.

In practice, we are interested in values of $\psi$ from irrigation channels which are not flat. From the results, regardless of the geometrical shapes of the irrigation channels, it appears that the value of $\psi$ is mostly affected by the width and the surface area of the channels. Since trapezoidal channels are commonly used more than the other type of channels, we specifically discuss infiltration from trapezoidal channels in different types of homogeneous soils. Touchet silt loam (TSL) and Guelph loam (GL) are considered besides PCL. For TSL, the values of $\alpha$ and $K_{s}$ are $0.0156 \mathrm{~cm}^{-1}$ and $41.99 \mathrm{~cm} \mathrm{~d}^{-1}$, respectively. For GL, $\alpha=0.034 \mathrm{~cm}^{-1}$ and $K_{s}=31.71 \mathrm{~cm} \mathrm{~d}^{-1}$. The values of $\psi$ at various values of $X$ are presented in Figure 3.

Figure 3 shows that soil with a higher value of $\alpha$ gives a higher value of $\psi$. This means that the water content in a coarser soil type is higher than in a finer type. This result is physically expected, as water infiltrates more rapidly in coarser soil. 
As before, $\psi$ decreases with $Z$ at $X=10 \mathrm{~cm}$ and $X=30 \mathrm{~cm}$, as seen in Figures 3(a) and (b). In contrast, $\psi$ increases with $Z$ at $X=70 \mathrm{~cm}$ and $X=90 \mathrm{~cm}$ (Figures 3(c) and (d)). It can also be seen that for different types of soil, the shapes of the graphs are preserved at the same value of $X$. This means that the difference in $\psi$ remains roughly a constant throughout the whole region.

\section{Concluding remarks}

A DRBEM has been formulated and successfully implemented for solving steadystate problems involving infiltration from four types of periodic channels. The method is applied to solve a type of Helmholtz equation numerically. Using the solutions obtained, numerical values of the suction potential are then computed. Moreover, infiltration from trapezoidal channels in three different types of homogeneous soil are also studied.

The results indicate that there is no significant difference in the suction potential for different types of nonflat periodic channels. However, the differences in the suction potential between the flat channel and the other types of channels are remarkable. For infiltration in different types of soil, the results imply that the suction potential in coarser soil is higher than in finer types.

Thus, for nonflat irrigation channels, the width and the surface area of the channels are the most important things to obtain desirable water content in the soil. In addition, if water availability in the soil is the most important factor in raising crops, then a coarser soil type is better than finer types.

\section{Acknowledgement}

Imam Solekhudin wishes to thank the Directorate General of the Higher Education of the Republic of Indonesia (DIKTI) for providing financial support for this research.

\section{References}

[1] A. Amoozegar-Fard, A. W. Warrick and D. O. Lomen, "Design nomographs for trickle irrigation systems", J. Irrigation Drainage Engrg. 110 (1984) 107-120; doi:10.1061/(ASCE)0733-9437(1984)110:2(107).

[2] W. T. Ang, "A Laplace transformation dual-reciprocity boundary element method for a class of two-dimensional microscale thermal problems", Engrg. Comput. 19 (2002) 467-478; doi:10.1108/02644400210430217.

[3] W. T. Ang and K. C. Ang, "A dual-reciprocity boundary element solution of a generalized nonlinear Schrödinger equation”, Numer. Methods Partial Differential Equations 20 (2004) 843-854; doi:10.1002/num.20011.

[4] M. I. Azis, D. L. Clements and M. Lobo, "A boundary element method for steady infiltration from periodic channels", ANZIAM J. 44 (2003) C61-C78; http://journal.austms.org.au/ojs/index.php/ANZIAMJ/article/view/672.

[5] V. Batu, "Steady infiltration from single and periodic strip sources", Soil Sci. Soc. Am. J. 42 (1978) 544-549; doi:10.2136/sssaj1978.03615995004200040033x.

[6] C. A. Brebbia and D. Nardini, "Dynamic analysis in solid mechanics by an alternative boundary element procedure", Int. J. Soil Dynam. Earthquake Engrg. 2 (1983) 228-233; doi:10.1016/0261-7277(83)90040-2. 
[7] E. Bresler, "Analysis of trickle irrigation with application to design problems", Irrigation Sci. 1 (1978) 3-17; doi:10.1007/BF00269003.

[8] D. L. Clements, M. Lobo and N. Widana, "A hypersingular boundary integral equation for a class of problems concerning infiltration from periodic channels", Electron. J. Bound. Elem. 5 (2007) 1-16; http://ejbe.libraries.rutgers.edu/index.php/ejbe/article/view/779.

[9] M. Lobo, D. L. Clements and N. Widana, "Infiltration from irrigation channels in a soil with impermeable inclusions", ANZIAM J. 46 (2005) C1055-C1068; http://journal.austms.org.au/ojs/index.php/ANZIAMJ/article/view/1006.

[10] J. R. Philip, "Flow in porous media", Annu. Rev. Fluid Mech. 2 (1970) 177-204; doi:10.1146/annurev.fl.02.010170.001141.

[11] A. J. Pullan, "Linearized time-dependent infiltration from a shallow pond", Water Resour. Res. 28 (1992) 1041-1046; doi:10.1029/91WR03032.

[12] R. T. Waechter and A. C. Mandal, "Steady infiltration from a semicircular cylindrical trench and a hemispherical pond into unsaturated soil”, Water Resour. Res. 29 (1993) 457-467; doi:10.1029/92WR02089.

[13] R. T. Waechter and J. R. Philip, "Steady two- and three-dimensional flows in unsaturated soil: the scattering analog", Water Resour. Res. 21 (1985) 1875-1887; doi:10.1029/WR021i012p01875.

[14] S. P. Zhu, P. Satravaha and X. P. Lu, "Solving linear diffusion equations with the dual reciprocity method in Laplace space", Eng. Anal. Bound. Elem. 13 (1994) 1-10;

doi:10.1016/0955-7997(94)90002-7. 